\title{
Occurrence and prognostic effect of cervical spine injuries and cervical artery injuries with concomitant severe head injury
}

\author{
Juho Vehviläinen ${ }^{1} \cdot$ Tuomas Brinck $^{2}$ • Matias Lindfors ${ }^{1}$ - Jussi Numminen ${ }^{3} \cdot$ Jari Siironen $^{1} \cdot$ Rahul Raj $^{1}$
}

Received: 6 November 2019 / Accepted: 27 February 2020 / Published online: 10 March 2020

(C) The Author(s) 2020

\begin{abstract}
Background Blunt cerebrovascular injuries (BCVIs) and cervical spinal injuries (CSIs) are not uncommon injuries in patients with severe head injury and may affect patient recovery. We aimed to assess the independent relationship between BCVI, CSI, and outcome in patients with severe head injury.

Methods We identified patients with severe head injury from the Helsinki Trauma Registry treated during 2015-2017 in a large level 1 trauma hospital. We assessed the association between BCVI and SCI using multivariable logistic regression, adjusting for injury severity. Our primary outcome was functional outcome at 6 months, and our secondary outcome was 6-month mortality. Results Of 255 patients with a cervical spine CT, 26 patients (10\%) had a CSI, and of 194 patients with cervical CT angiography, 16 patients $(8 \%)$ had a BCVI. Four of the 16 BCVI patients had a BCVI-related brain infarction, and four of the CSI patients had some form of spinal cord injury. After adjusting for injury severity in multivariable logistic regression analysis, BCVI associated with poor functional outcome (odds ratio $[\mathrm{OR}]=6.0,95 \% \mathrm{CI}$ [confidence intervals] $=1.4-26.5)$ and mortality $(\mathrm{OR}=7.9,95 \% \mathrm{CI}$ 2.0-31.4). We did not find any association between CSI and outcome.

Conclusions We found that BCVI with concomitant head injury was an independent predictor of poor outcome in patients with severe head injury, but we found no association between CSI and outcome after severe head injury. Whether the association between BCVI and poor outcome is an indirect marker of a more severe injury or a result of treatment needs further investigations.
\end{abstract}

Keywords Head injury · Traumatic brain injury $\cdot$ Blunt cerebrovascular injury $\cdot$ Cervical spine injury $\cdot$ Cervical trauma

\section{Introduction}

Blunt cerebrovascular injuries (BCVIs) are a nonpenetrating injury to the carotid and/or vertebral artery that may cause stroke in trauma patients [5]. The

This article is part of the Topical Collection on Brain trauma

Electronic supplementary material The online version of this article (https://doi.org/10.1007/s00701-020-04279-9) contains supplementary material, which is available to authorized users.

Rahul Raj

Rahul.raj@hus.fi

1 Department of Neurosurgery, University of Helsinki and Helsinki University Hospital, Topeliuksenkatu 5, PO. Box 266, 00029 Helsinki, Finland

2 Department of Orthopaedics and Traumatology, University of Helsinki and Helsinki University Hospital, Helsinki, Finland

3 Department of Radiology, University of Helsinki and Helsinki University Hospital, Helsinki, Finland occurrence of BCVI in trauma patients has varied in literature between 0.08 and $1.2 \%[1,30,36,40]$. Higher $(9.2 \%)$ occurrence among traumatic brain injury (TBI) patients has been reported [12]. There have been also findings that a lack of adequate imaging techniques could give too low incidence and incidences of 2.7-6.7\% among trauma patients have been reported [4, 13].

The occurrence of cervical spine injuries (CSIs) ranges between 4 and $12.5 \%$ in TBI patients $[4,11,13,16,17,19$, $24,38,40,41]$. The overall occurrence of CSI in trauma patients is around 4\% [19]. Carotid artery injuries (CAI) and vertebral artery injuries (VAI) seem to be different entities in terms of risk factors and patient outcomes. CSI correlates to VAI in cervical hyperextension injuries at high impact energies [34]. Up to 70-78\% of VAI patients have a simultaneous CSI [3, 8, 34]. Correlating injuries and predisposing conditions to $\mathrm{CAI}$ are facial injuries, diffuse axonal injury (DAI), Glasgow Coma Scale $(\mathrm{GCS})<6$, and road traffic collisions $[34,40]$. In terms of patient outcome, CAI seem to be worse than VAI [10]. 
Stroke rate among BCVI patients has a large variation from $1 \%$ up to $53.8 \%$. The majority of these strokes were already in progress when the patient has been admitted to a hospital [5, $10,12,17,26,30,39,40,42]$.

Treatment of BCVI using either antiplatelet or anticoagulation medication seems to be equal [10]. However, there seems to be indications that the stroke rate in BCVI patients is around $10 \%$ in average regardless of any kind of intervention. This supports the theory that most of the strokes due to BCVI have already realized before admission to hospital $[17,26]$.

There have been efforts to construct evidence-based guidelines for treating BCVI patients [5, 9, 31]. According to guidelines, the indication criteria for computed tomography angiography (CTA) of cervical arteries would use extended Denver protocol, which includes signs and symptoms for BCVI, e.g., focal neurological deficit, expanding cervical hematoma, neurological deficit inconsistent of head computed tomography (CT), and stroke on CT/magnetic resonance imaging (MRI), or risk factors of BCVI, e.g., high-energy trauma, facial fractures, CSI, and TBI with thoracic injuries [9, 13, 14]. If these conditions are satisfied, the patient should go through CT angiography, and if BCVI is found, an antithrombotic treatment should be started as early as possible for minimum of 3 months. In addition, after 1 week and 3 months, a CT angiography control should be done [5]. Still, it seems that more rigorous screening of BCVI might not help. Even though more BCVI cases were found, it did not improve patient prognosis [18]. Also, a high number, up to $45 \%$, of false positive BCVI in CTA have been reported in initial imaging [35].

The aims of this study were to investigate the association between cervical BCVI, CSI, and outcome in patients with severe head injury. Furthermore, we aimed to establish the occurrence of cervical BCVI and CSI in patients with severe head injury treated in the largest Finnish trauma center. We hypothesized that the presence of a cervical BCVI or CSI would not affect outcome after severe head injury and that the occurrence of cervical BCVI and CSI in this Finnish cohort of severe head injury patients would be lesser than in previously reported studies from settings outside the Nordics. The rationale for this hypothesis is that severe head injuries in Finland are often the result of low-energy trauma mechanisms among elderly patients $[27,33]$.

\section{Methods and materials}

\section{Study setting and study population}

We conducted a retrospective study of the prospectively data collecting Helsinki Trauma Registry (HTR), which has been described in detail previously [21]. The local research committee approved of the study (HUS/175/2016). We obtained through the HTR data on severely injured patients, New Injury Severity Score (NISS) $>15$, treated from May 2015 through May 2017. HTR is a local trauma registry in the trauma unit of the Helsinki University Hospital, which centralizes the treatment of severe blunt injuries among adult patients ( $\geq 16$ years) with a catchment area of 1.8 million inhabitants in southern Finland.

The trauma unit of Helsinki University Hospital implemented a routine contrast $\mathrm{CT}$ imaging of cervical vessels of trauma alert patients in May 2015. This allowed us to investigate the occurrence of blunt vascular trauma in the cervical area in addition to the lesions in cervical spine.

We included patients with a severe head injury (head Abbreviated Injury Score (AIS) $\geq 3$ ) [15]. We screened all patients who underwent a whole-body contrast CT including the cervical arteries and all patients who underwent a noncontrast CT scan of the cervical spine.

\section{Definition of covariates}

We assessed the severity of the CSI according to the AIS classification [15]. We further divided those with CSI patients into two groups: surgically treated and non-surgically treated CSI.

We used the Biffl grades for classification of cervical BCVI [2]. All cervical artery $C T$ angiograms were primarily assessed by specialized radiologists. We checked the reports of all scans and re-reviewed (authors JN and RR) those with a confirmed or suspected cervical BCVI. If a lesion in cervical arteries was found, later head CT or/and MRI scans of head were screened for cerebral ischemia. We defined a brain infarction if there were signs of a new ischemic lesion in the territory of the affected cervical artery.

We defined the GCS score as the first reliable postresuscitation GCS score prior to intubation or sedation. We defined hypotension and hypoxia as a prehospital, admission, or emergency room systolic blood pressure $<90 \mathrm{mmHg}$ and an oxygen saturation $<90 \%$. The head CT images were analyzed using Marshall CT classification [28]; in addition, epidural hematomas (EDH) and traumatic subarachnoid hemorrhages $(\mathrm{tSAH})$ were identified in the images.

\section{Definition of outcomes}

Our aim was to assess the association between cervical BCVI, CSI, and outcome after severe head injury. Our primary outcome was 6-month functional outcome, and our secondary outcome was 6-month mortality. We defined functional outcome according to the Glasgow Outcome Score (GOS) [25]. We assessed GOS from various electronic health care records (including neurosurgical and neurological follow-up visits, rehabilitation visits, neuropsychological visits, primary health care visits) retrospectively at 6 months' time of the initial 
injury. We defined a GOS of 1-3 (death to severe disability) as unfavorable outcome and a GOS of 4-5 (moderate disability to good recovery) as favorable outcome. Data regarding 6month mortality was obtained through the population registry and available for all patients.

\section{Statistical analyses}

We used IBM SPSS Statistics version 24 for Windows (IBM Corp.) for the statistical analyses. Categorical variables are compared using a two-sided $\chi^{2}$ test. Continuous data were tested for skewness using the Kolmogorov-Smirnov test. Non-parametric data were compared using a Mann-Whitney $U$ test, and normally distributed data were compared using an independent $t$ test.

To assess the independent association between cervical BCVI and CSI, we adjusted for brain injury severity, using an IMPACT extended-based model. We included all predictors from the IMPACT extended model and calibrated the predictors' coefficients to our dataset using multivariable logistic regression (LR) with 6-month outcome as the outcome. We assessed the area under the receiver operating curve (AUC) to assess the performance of the IMPACT extended model to adjust for case-mix. We then used the IMPACT extended model to adjust for injury severity in separate multivariable LR model with cervical BCVI and CSI as separate predictors.

Regarding missing data, we used a multiple imputation with fully conditional specification Markov Chain Monte Carlo (MCMC) method with 10 iterations. The IMPACT extended predictors that had missing values were hypoxia, hypotension, and pupil light reaction. The predictors for the MCMC analysis were age, Marshall CT classification, epidural hematoma, traumatic SAH, systolic blood pressure, oxygen saturation, NISS, and death within 30 days. Little's MCAR test yielded a $p$ value of 0.65 , indicating randomly missing data.

\section{Results}

\section{Baseline characteristics}

A total of 280 patients with severe head injury were identified from the HTR during the study period (Fig. 1). Of these, 255 patients $(91 \%)$ had a cervical spine CT performed and 194 patients $(69 \%)$ had a cervical CT angiography performed (all patients with a cervical CTA had also a cervical spine CT performed).

Table 1 shows the patient demographics in patients who underwent $\mathrm{CT}$ of the cervical spine. Differences between those with and without CSI were found in injury type (traffic accidents have the highest CSI occurrence),and Marshall CT classification.

Table 2 shows the patient demographics in patients who underwent a CTA of the cervical arteries. There were no statistically significant differences in baseline characteristics between those with and without BCVI.

Online Resource 1 describes the patient demographics for the whole dataset $(N=280)$ and for sub-groups in which the cervical spine $(N=25)$ or the cervical arteries $(N=86)$ were not imaged. The main difference between the whole dataset and the not-imaged sub-groups was that patients in notimaged sub-groups had more frequently low energy traumas.

Online Resource 2 describes the mean values of the nonimputed and imputed datasets. The imputation did not notably affect the mean values of the imputed variables.

\section{Cervical spine injury}

Of the 255 patients who underwent $\mathrm{CT}$ of the cervical spine, 26 patients (10\%) were diagnosed with a CSI. Four out of 26 CSI patients (15\%) underwent operative treatment. Four CSI patients had varying types of spinal cord injuries ranging from small epidural hematoma to complete cord injury. One patient with complete cord injury and BCVI (Biffl grade 4) died. One patient had a favorable neurological outcome, two patients were from different hospital districts, and no follow-up neurological outcome information was available. These two patients were, however, alive within 6 months of the injury.

\section{Blunt cervical vascular injury}

Of the 194 patients who underwent CTA of the cervical arteries, BCVI was found in 16 patients (8\%, Online Resource 3). Almost two thirds of the BCVI were in the carotid arteries. There were a total of four BCVI-related brain infarctions (all carotid artery). Three out of 4 patients with a BCVI-related brain infarction died within 6 months, and the remaining patient had an unfavorable functional outcome (Online Resource $3)$.

\section{Multivariable analysis}

The AUC for the IMPACT extended model for predicting 6-month mortality was $0.90-0.91$ (95\% CI $0.86-0.96$, depending on the imputed dataset), and the AUC for predicting 6-month functional status was 0.86 (95\% CI 0.81-0.91, depending on the imputed dataset), thus providing excellent case-mix adjustment.

After adjusting for case-mix, the presence of a CSI did not associate with 6-month unfavorable outcome $(\mathrm{OR}=2.09$, $95 \%$ CI $0.67-6.54, p=0.205$ ) or 6-month mortality ( $\mathrm{OR}=$ $1.89,95 \%$ CI $0.49-7.33, p=0.358)$. 


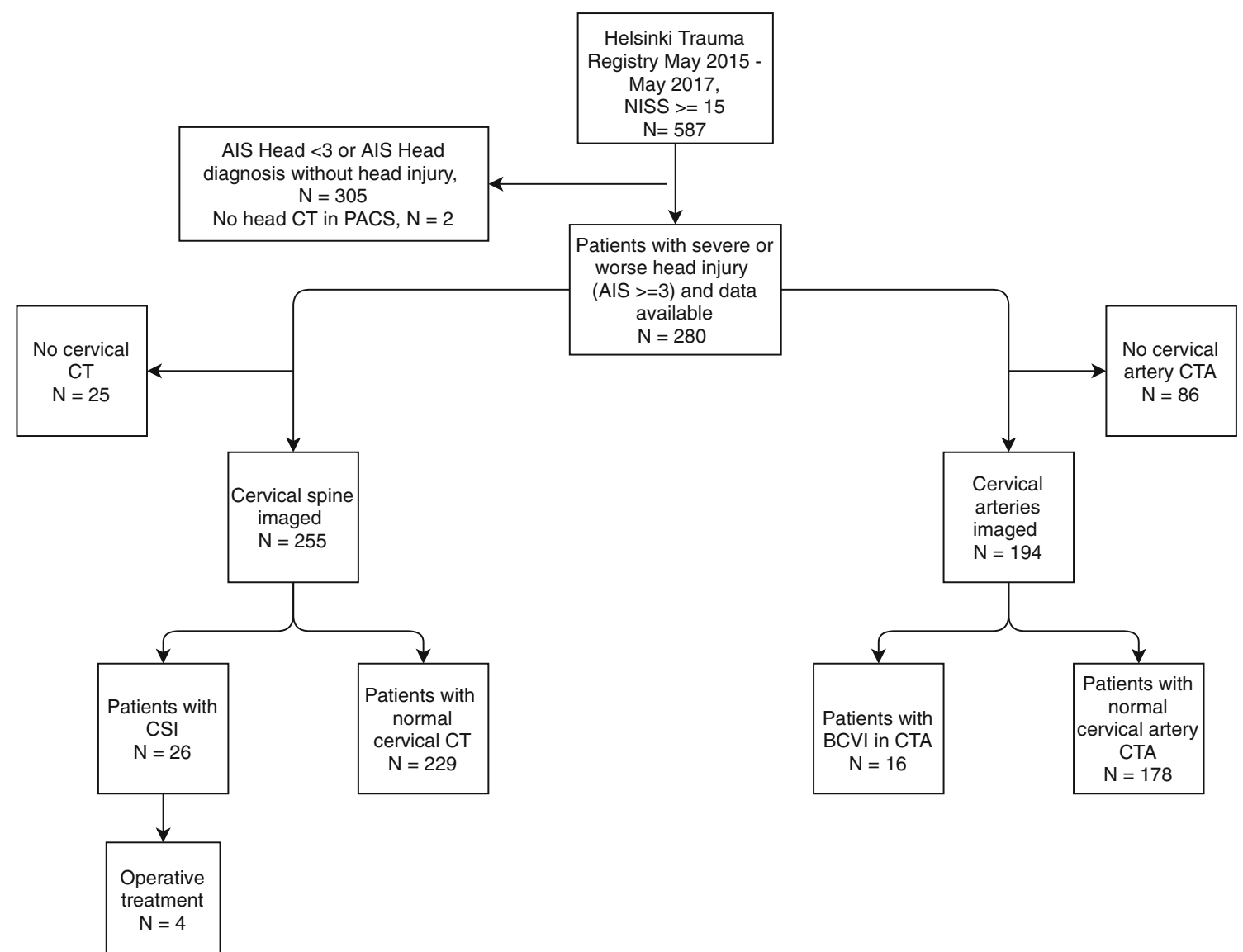

Fig. 1 Flow chart of the patients in the study. AIS Head $\geq 3$ is defined as severe head trauma. AIS, Abbreviated Injury Score; BCVI, blunt cerebrovascular injury; CSI, cervical spine injury; CT, computed

tomography; CTA, computed tomography angiography; NISS, New Injury Severity Score; PACS, picture archiving and communication systems

After adjusting for case-mix, the presence of a BCVI significantly associated with 6-month unfavorable outcome $(\mathrm{OR}=5.99,95 \% \mathrm{CI} 1.35-26.51, p=0.018)$ and 6-month mortality $(\mathrm{OR}=7.90,95 \%$ CI 1.99-31.43, $p=0.003)$.

\section{Discussion}

In this retrospective study investigating the association between CSI, BCVI, and outcome in patients with severe head injury, we found that BCVI with concomitant severe head injury was associated with unfavorable outcome and adds to 6-month mortality. CSI on the other hand did not affect patient outcome or 6-month mortality. Of included patients, $8.2 \%$ had a diagnosed BCVI and $10.2 \%$ a diagnosed CSI. Thus, contrary to our hypothesis, the occurrences of BCVI and CSI were rather similar in our setting compared to other settings $[12,16]$.

Also, contrary to our hypothesis, we found that BCVI was associated with an approximately 6 times higher odds for poor functional outcome and 8 times higher odds for 6-month mortality. Still, there was no difference in the severity of brain

injury (according to the IMPACT extended model) between those with and without BCVI.

There are several reasons why BCVI may lead to poorer outcome after severe head injury. BCVI-related infarcts are the obvious reason. In our cohort, four out of 16 patients had a diagnosed BCVI-related infarction, all of which had a poor outcome. Due to the skewed distribution of BCVI in the outcome groups (all patients with BCVI-related infarct had an unfavorable outcome), it was not possible to assess the independent association between BCVI-related brain infarction and outcome. Yet, it is plausible to relate these infarcts to poorer outcome. Still, one should notice that half of the patients with a BCVI-related infarct underwent MRI. It is possible that whether all patients would have undergone brain MRI scans, the occurrence of BCVI-related brain infarcts would have been higher. It is also possible that the BCVI patients received earlier and more aggressive antithrombotic medication therapies, which may increase the risk of intracranial hemorrhage progression. For 12 BCVI patients, low molecular weight heparin (LMWH) was initiated on post-traumatic days 1 to 5, and for four BCVI patients, LMWH could not or had not been initiated due to hemorrhage progression and/or 
Table 1 Patient demographics for all 255 patients who underwent a CT of the cervical spine according to the presence of cervical spine injury status

\begin{tabular}{|c|c|c|c|c|}
\hline Variable & All CT spine $(N=255)$ & $\mathrm{CSI}(N=26)$ & No CSI $(N=229)$ & $p$ value \\
\hline Age, median (IQR) & $52(35-66)$ & $45(35-55)$ & $53(35-67)$ & 0.094 \\
\hline Gender ( $\%$ males $)$ & 76 & 85 & 75 & 0.282 \\
\hline \multicolumn{4}{|l|}{ ASA } & 0.169 \\
\hline 1 & $97(38 \%)$ & $13(50 \%)$ & $84(37 \%)$ & \\
\hline 2 & $104(41 \%)$ & $12(46 \%)$ & $92(40 \%)$ & \\
\hline 3 & $46(18 \%)$ & $1(4 \%)$ & $45(20 \%)$ & \\
\hline 4 & $5(2 \%)$ & 0 & $5(2 \%)$ & \\
\hline Missing & $3(1 \%)$ & 0 & $3(1 \%)$ & \\
\hline \multicolumn{4}{|l|}{ Injury type } & $0.022 *$ \\
\hline High fall & $45(18 \%)$ & $4(15 \%)$ & $41(18 \%)$ & \\
\hline Low fall & $69(27 \%)$ & $1(4 \%)$ & $68(30 \%)$ & \\
\hline Traffic, motor vehicle & $47(18 \%)$ & $9(35 \%)$ & $38(17 \%)$ & \\
\hline Traffic, bicycle & $21(8 \%)$ & $5(19 \%)$ & $16(7 \%)$ & \\
\hline Traffic, pedestrian & $13(5 \%)$ & $3(12 \%)$ & $10(4 \%)$ & \\
\hline Other & $27(11 \%)$ & $1(4 \%)$ & $26(11 \%)$ & \\
\hline Unknown & $33(13 \%)$ & $3(12 \%)$ & $30(13 \%)$ & \\
\hline \multicolumn{4}{|l|}{ GCS } & 0.730 \\
\hline $3-8$ & $131(51 \%)$ & $13(50 \%)$ & $118(52 \%)$ & \\
\hline $9-13$ & $55(22 \%)$ & $3(12 \%)$ & $52(23 \%)$ & \\
\hline $14-15$ & $62(24 \%)$ & $8(31 \%)$ & $54(24 \%)$ & \\
\hline Missing & $7(3 \%)$ & $2(8 \%)$ & $5(2 \%)$ & \\
\hline \multicolumn{4}{|l|}{ Motor score } & 0.297 \\
\hline $1-2$ & $89(35 \%)$ & $7(27 \%)$ & $82(36 \%)$ & \\
\hline $3-4$ & $37(15 \%)$ & $6(23 \%)$ & $31(14 \%)$ & \\
\hline $5-6$ & $118(46 \%)$ & $11(42 \%)$ & $107(47 \%)$ & \\
\hline Missing & $11(4 \%)$ & $2(8 \%)$ & $9(4 \%)$ & \\
\hline \multicolumn{4}{|l|}{ Pupils } & 0.356 \\
\hline Responsive & $138(54 \%)$ & $18(69 \%)$ & $120(52 \%)$ & \\
\hline Unilateral unresponsive & $17(7 \%)$ & $1(4 \%)$ & $16(7 \%)$ & \\
\hline Bilateral unresponsive & $69(27 \%)$ & $5(19 \%)$ & $64(28 \%)$ & \\
\hline Missing & $31(12 \%)$ & $2(8 \%)$ & $29(13 \%)$ & \\
\hline \multicolumn{5}{|l|}{ Cardio-pulmonary system } \\
\hline Hypotension & $17(7 \%)$ & $3(12 \%)$ & $14(6 \%)$ & 0.280 \\
\hline Missing & $6(2 \%)$ & $1(4 \%)$ & $5(2 \%)$ & \\
\hline Hypoxia & $7(3 \%)$ & $1(4 \%)$ & $7(3 \%)$ & 0.369 \\
\hline Missing & $7(3 \%)$ & 0 & $6(3 \%)$ & \\
\hline \multicolumn{4}{|l|}{ Marshall CT classification } & $0.003 *$ \\
\hline I & $16(6 \%)$ & $3(12 \%)$ & $13(6 \%)$ & \\
\hline II & $124(49 \%)$ & $19(73 \%)$ & $105(46 \%)$ & \\
\hline III & $15(6 \%)$ & $3(12 \%)$ & $12(5 \%)$ & \\
\hline IV & $15(6 \%)$ & 0 & $15(7 \%)$ & \\
\hline $\mathrm{V}+\mathrm{VI}$ & $85(33 \%)$ & $1(4 \%)$ & $84(37 \%)$ & \\
\hline $\mathrm{tSAH}$ & $152(60 \%)$ & $16(62 \%)$ & $136(59 \%)$ & 0.832 \\
\hline $\mathrm{EDH}$ & $32(13 \%)$ & $1(4 \%)$ & $31(14 \%)$ & 0.157 \\
\hline IMPACT extended risk for 6-month mortality (IQR) & $39.7 \%(19.1-75.3 \%)$ & $23.4 \%(16.4-60.0 \%)$ & $45.9 \%(19.1-79.8 \%)$ & 0.094 \\
\hline IMPACT extended risk for 6-month unfavorable outcome (IQR) & $65.9 \%(39.1-91.0 \%)$ & $45.8 \%(34.3-82.0 \%)$ & $71.8 \%(39.1-93.0 \%)$ & 0.094 \\
\hline Missing values for IMPACT calculation & $37(15 \%)$ & $2(8 \%)$ & $35(15 \%)$ & \\
\hline NISS, median (IQR) & $33.0(26.0-43.0)$ & $34.0(24.5-39.5)$ & $33.0(26.0-43.0)$ & 0.610 \\
\hline \multicolumn{4}{|l|}{ Outcome 6 months } & 0.401 \\
\hline Dead & $62(24 \%)$ & $5(19 \%)$ & $57(25 \%)$ & \\
\hline GOS 2-3 & $37(15 \%)$ & $4(15 \%)$ & $33(14 \%)$ & \\
\hline GOS 4-5 & $123(48 \%)$ & $12(46 \%)$ & $111(48 \%)$ & \\
\hline Missing & $33(13 \%)$ & $5(19 \%)$ & $28(12 \%)$ & \\
\hline
\end{tabular}

Abbreviations: CSI cervical spine injury, CT computed tomography, CTA computed tomography angiography, GCS Glasgow Coma Score, tSAH traumatic subarachnoidal hemorrhage, EDH epidural hematoma, NISS New Injury Severity Score, GOS Glasgow Outcome Score, Hypotension systolic $\mathrm{BP}<90$, Hypoxia $\mathrm{SpO} 2<90 \%$. If GCS or pupil response was missing at the time of hospital admission, prehospital values were used

$*$ Statistically significant $p<0.05$

dismal prognosis. Out of the four BCVI patients with a diagnosed brain infarction, LMWH was initiated on post- traumatic days 1 to 2 for two patients, and for two patients, LMWH was not initiated due to the latter aforementioned 
Table 2 Patient demographics for all 194 patients who underwent a CT angiography of the cervical arteries according to the presence of blunt cervical vascular injury (BCVI)

\begin{tabular}{|c|c|c|c|c|}
\hline & All CT cervical arteries $(N=194)$ & $\operatorname{BCVI}(N=16)$ & No BCVI $(N=178)$ & $p$ value \\
\hline Age, median (IQR) & $48(32-64)$ & $50(32-62)$ & $48(32-65)$ & 0.773 \\
\hline Gender ( $\%$ males) & 76 & 88 & 75 & 0.253 \\
\hline \multicolumn{4}{|l|}{ ASA } & 0.701 \\
\hline 1 & $86(44 \%)$ & $9(56 \%)$ & $77(43 \%)$ & \\
\hline 2 & $77(40 \%)$ & $6(38 \%)$ & $71(40 \%)$ & \\
\hline 3 & $25(13 \%)$ & $1(6 \%)$ & $24(14 \%)$ & \\
\hline 4 & $3(2 \%)$ & 0 & $3(2 \%)$ & \\
\hline Missing & $3(2 \%)$ & 0 & $3(2 \%)$ & \\
\hline \multicolumn{4}{|l|}{ Injury type } & 0.388 \\
\hline High fall & $41(21 \%)$ & $3(19 \%)$ & $38(21 \%)$ & \\
\hline Low fall & $29(15 \%)$ & $1(6 \%)$ & $28(16 \%)$ & \\
\hline Traffic, motor vehicle & $47(24 \%)$ & $6(38 \%)$ & $41(23 \%)$ & \\
\hline Traffic, bicycle & $21(11 \%)$ & $3(19 \%)$ & $18(10 \%)$ & \\
\hline Traffic, pedestrian & $12(6 \%)$ & $2(13 \%)$ & $10(6 \%)$ & \\
\hline Other & $20(10 \%)$ & 0 & $19(11 \%)$ & \\
\hline Unknown & $24(12 \%)$ & $1(6 \%)$ & $23(13 \%)$ & \\
\hline \multicolumn{4}{|l|}{ GCS } & 0.559 \\
\hline $3-8$ & $98(51 \%)$ & $10(63 \%)$ & $88(49 \%)$ & \\
\hline $9-13$ & $42(22 \%)$ & $2(13 \%)$ & $40(22 \%)$ & \\
\hline $14-15$ & $49(25 \%)$ & $3(19 \%)$ & $46(26 \%)$ & \\
\hline Missing & $5(3 \%)$ & $1(6 \%)$ & $4(2 \%)$ & \\
\hline \multicolumn{4}{|l|}{ Motor score } & 0.328 \\
\hline $1-2$ & $70(36 \%)$ & $9(56 \%)$ & $61(34 \%)$ & \\
\hline $3-4$ & $25(13 \%)$ & $1(6 \%)$ & $24(13 \%)$ & \\
\hline $5-6$ & $90(46 \%)$ & $5(31 \%)$ & $85(48 \%)$ & \\
\hline Missing & $9(5 \%)$ & $1(6 \%)$ & $8(5 \%)$ & \\
\hline \multicolumn{4}{|l|}{ Pupils } & 0.562 \\
\hline Responsive & $112(58 \%)$ & $11(69 \%)$ & $101(57 \%)$ & \\
\hline Unilateral unresponsive & $15(8 \%)$ & $1(6 \%)$ & $14(8 \%)$ & \\
\hline Bilateral unresponsive & $43(22 \%)$ & $2(13 \%)$ & $41(23 \%)$ & \\
\hline Missing & $24(12 \%)$ & $2(13 \%)$ & $22(12 \%)$ & \\
\hline \multicolumn{5}{|l|}{ Cardio-pulmonary system } \\
\hline Hypotension & $15(8 \%)$ & $3(19 \%)$ & $12(7 \%)$ & 0.067 \\
\hline Missing & $2(1 \%)$ & $1(6 \%)$ & $1(1 \%)$ & \\
\hline Нypoxia & $37(19 \%)$ & $4(25 \%)$ & $33(19 \%)$ & 0.637 \\
\hline Missing & $21(11 \%)$ & $3(19 \%)$ & $18(10 \%)$ & \\
\hline \multicolumn{4}{|l|}{ Marshall CT classification } & 0.432 \\
\hline I & $16(8 \%)$ & $2(13 \%)$ & $14(8 \%)$ & \\
\hline II & $113(58 \%)$ & $9(56 \%)$ & $104(58 \%)$ & \\
\hline III & $13(7 \%)$ & $2(13 \%)$ & $11(6 \%)$ & \\
\hline IV & $13(7 \%)$ & $2(13 \%)$ & $11(6 \%)$ & \\
\hline $\mathrm{V}+\mathrm{VI}$ & $39(20 \%)$ & $1(6 \%)$ & $38(21 \%)$ & \\
\hline $\mathrm{tSAH}$ & $115(59 \%)$ & $12(75 \%)$ & $103(58 \%)$ & 0.181 \\
\hline $\mathrm{EDH}$ & $23(12 \%)$ & $1(6 \%)$ & $22(12 \%)$ & 0.469 \\
\hline IMPACT extended risk for 6-month mortality (IQR) & $33.7 \%(13.9-70.3 \%)$ & $\begin{array}{l}55.3 \% \\
\quad(10.8-80.7 \%)\end{array}$ & $\begin{array}{l}33.7 \% \\
\quad(15.5-70.3 \%)\end{array}$ & 0.368 \\
\hline IMPACT extended risk for 6-month unfavorable outcome (IQR) & $59.5 \%(29.9-88.5 \%)$ & $\begin{array}{l}78.6 \% \\
\quad(23.7-93.4 \%)\end{array}$ & $\begin{array}{l}59.5 \% \\
\quad(32.7-88.5 \%)\end{array}$ & 0.368 \\
\hline Missing values for IMPACT calculation & $25(13 \%)$ & $2(13 \%)$ & $23(13 \%)$ & \\
\hline NISS, median (IQR) & $33.5(27.0-43.0)$ & $38.0(27.0-43.0)$ & $33.0(26.0-43.0)$ & 0.136 \\
\hline \multicolumn{4}{|l|}{ Outcome 6 months } & 0.053 \\
\hline Dead & $41(21 \%)$ & $8(50 \%)$ & $33(19 \%)$ & \\
\hline GOS $2-3$ & $26(13 \%)$ & $3(19 \%)$ & $23(13 \%)$ & \\
\hline GOS 4-5 & $101(52 \%)$ & $4(25 \%)$ & $97(54 \%)$ & \\
\hline Missing & $26(13 \%)$ & $1(6 \%)$ & $25(14 \%)$ & \\
\hline
\end{tabular}

Abbreviations: CSI cervical spine injury, CT computed tomography, CTA computed tomography angiography, GCS Glasgow Coma Score, $t S A H$ traumatic subarachnoidal hemorrhage, EDH epidural hematoma, NISS New Injury Severity Score, GOS Glasgow Outcome Score, Hypotension systolic $\mathrm{BP}<90$, Hypoxia $\mathrm{SpO} 2<90 \%$. If GCS or pupil response was missing at the time of hospital admission, prehospital values were used

*Statistically significant $p<0.05$ 
reason. Importantly, patients with BCVI were more often subject to high-energy traumas. Thus, it is likely that patients with concomitant severe head injury and BCVI really had more severe injuries than those without a BCVI, despite of similar IMPACT extended model predictions, which might fail to adjust for the full spectrum of differences in injury severity.

According to our hypothesis, we did not find any association between CSI and outcome. Again, there were no differences in injury severity as measured by the IMPACT extended model between those with and without a CSI. Like the BCVI patients, those with CSI were more frequently in a highenergy trauma than those without CSI. However, this did not reflect in poorer outcomes. Of those with CSI, only four had a documented spinal cord injury. It is possible that CSI may have been an indirect marker of poor outcome, whether the prevalence of spinal cord injury would have been higher. Other studies have found that those with CSI and spinal cord injury have poorer outcomes than those CSI without spinal cord injury, which is in line with our findings [20,32].

Currently, there are evidence-based guidelines for the treatment of spontaneous carotid dissections [6, 29]; however, in terms of BCVI, the treatment guidelines are still in progress. The choice between antiplatelet versus anticoagulation and the time for optimal medication start remain unknown. Based on this retrospective series, it is not possible to draw any conclusions but only to highlight the relatively strong association between BCVI and outcome. Whether this is due to the BCVI itself, higher trauma energies and more severe injuries, or possible negative effects of the treatment requires further studies, and due to the relative rareness of BCVI, probably multicenter collaborative projects.

Regarding the occurrence of BCVI, likely, the most widespread recommendation to screen for BCVI is the extended Denver protocol $[9,13,14]$. Other screening recommendations are, for example, modified Memphis criteria [7]. For CSI, the de facto screening criteria have been the National Emergency X-Radiography Utilization Study (NEXUS) criteria and the Canadian C-spine rules [22, 23, 37]. The local protocol used in HTR is in accordance with these screening protocols, and the occurrence of BCVI and CSI in our cohort was similar to that of previous studies $(9.2 \%$ for BCVI [12] and $4-12.5 \%$ for CSI $[4,11,13,16,17,19,24,38,40,41])$, indicating appropriate usefulness of the screening guidelines.

\section{Strengths and limitations}

This study is a cohort study including consecutive patients admitted to the trauma unit of Helsinki University Hospital (catchment area of almost 2 million for severe head injury), which is one of the largest trauma units in the Nordic countries. Despite our study being retrospective in nature, the high-quality electronic patient registries enable excellent follow-up in combination with extensive and validated data from the HTR [21].

Still, there are limitations that should be addressed. First, we had a relatively small number of both BCVIs and CSIs. Thus, the statistical analyses may be somewhat underpowered (e.g., association between BCVI-related brain infarction and outcome). Second, not all patients underwent cervical CT or CTA. Thus, we were forced to separate these groups from one another. Still, there were no major differences in patient characteristics between those with and without complete imaging studies. Thus, we do not believe that this affects our results. Third, we do not routinely screen BCVI patients for brain infarcts using MRI. Still, eight out of 16 patients with a BCVI underwent a brain MRI at some point after the trauma. Thus, it is possible that clinically silent infarcts may have been undiagnosed, underestimating the occurrence of BCVI-related infarcts. Third, although CTA of the cervical arteries and CT of the spine were part of the routine trauma protocol for severe head injury patients during the study period, $9 \%$ of patients did not undergo a cervical spine CT and $31 \%$ of patients did not undergo a CTA of the cervical arteries. Reasons for protocol deviations require further investigation. Fourth, the possibility of false positives for diagnosing BCVI has been described to be as high as $45 \%$ [35]. We re-reviewed the images of patients with a radiological or clinical suspicion of BCVI. Thus, it is possible that there were patients with an initially misdiagnosed or silent BCVI that we did not pick up. However, whether these misdiagnosed or silent BCVI are clinically meaningful remains unknown, but we do not believe this to affect our results.

\section{Summary}

We found that BCVI with concomitant head injury was an independent predictor of poor outcome in patients with severe head injury, but we found no association between CSI and outcome after severe head injury. The occurrence of BCVI in our study was $8 \%$ that is similar to previous studies in patients with severe head injury [12]. Whether the association between BCVI and poor outcome is an indirect marker of a more severe injury or a result of treatment needs further investigations.

Funding information Open Access funding provided by niversity of Helsinki including Helsinki University Central Hospital. Independent research funding support has been received from Helsinki University Hospital (RR, JV), Finska Läkaresällskapet (RR), Medicinska Understödsföreningen Liv \& Hälsa (RR), and Svenska Kulturfonden (RR). The funders had no role in the study design, data collection, data analysis, data interpretation, or writing of the manuscript. The first and last authors had full access to all the data in the study and had final responsibility for the decision to submit for publication. 


\section{Compliance with ethical standards}

Conflict of interest The authors declare that they have no conflict of interest.

Ethical approval All procedures performed in studies involving human participants were in accordance with the ethical standards of the institutional and/or national research committee (HUS/175/2016) and with the 1964 Helsinki Declaration and its later amendments or comparable ethical standards. For this type of study, formal consent is not required.

Open Access This article is licensed under a Creative Commons Attribution 4.0 International License, which permits use, sharing, adaptation, distribution and reproduction in any medium or format, as long as you give appropriate credit to the original author(s) and the source, provide a link to the Creative Commons licence, and indicate if changes were made. The images or other third party material in this article are included in the article's Creative Commons licence, unless indicated otherwise in a credit line to the material. If material is not included in the article's Creative Commons licence and your intended use is not permitted by statutory regulation or exceeds the permitted use, you will need to obtain permission directly from the copyright holder. To view a copy of this licence, visit http://creativecommons.org/licenses/by/4.0/.

\section{References}

1. Berne JD, Norwood SH, McAuley CE, Vallina VL, Creath RG, McLarty J (2001) The high morbidity of blunt cerebrovascular injury in an unscreened population: more evidence of the need for mandatory screening protocols11 No competing interests declared. J Am Coll Surg 192(3):314-321

2. Biffl WL, Moore EE, Offner PJ, Brega KE, Franciose RJ, Burch JM (1999) Blunt carotid arterial injuries: implications of a new grading scale. J Trauma 47(5):845-853

3. Biffl WL, Egglin T, Benedetto B, Gibbs F, Cioffi WG (2006) Sixteen-slice computed tomographic angiography is a reliable noninvasive screening test for clinically significant blunt cerebrovascular injuries. J Trauma Injury Infect Crit Care 60(4):745-752

4. Borisch I, Boehme T, Butz B, Hamer OW, Feuerbach S, Zorger N (2007) Screening for carotid injury in trauma patients: image quality of 16-detector-row computed tomography angiography. Acta Radiol 48(7):798-805

5. Brommeland T, Helseth E, Aarhus M, Moen KG, Dyrskog S, Bergholt B, Olivecrona Z, Jeppesen E (2018) Best practice guidelines for blunt cerebrovascular injury (BCVI). Scandinavian Journal of Trauma, Resuscitation and Emergency Medicine 26(1): 90

6. Brott TG, Halperin JL, Abbara S et al (2011) 2011 ASA/ACCF/ AHA/AANN/AANS/ACR/ASNR/CNS/SAIP/SCAI/SIR/SNIS/ SVM/SVS guideline on the management of patients with extracranial carotid and vertebral artery disease. Circulation. https://doi.org/ 10.1161/CIR.0b013e31820d8c98

7. Ciapetti M, Circelli A, Zagli G, Migliaccio ML, Spina R, Alessi A, Acquafresca M, Bartolini M, Peris A (2010) Diagnosis of carotid arterial injury in major trauma using a modification of Memphis criteria. Scand J Trauma Resusc Emerg Med. https://doi.org/10. 1186/1757-7241-18-61

8. Cothren CC, Moore EE, Biffl WL, Ciesla DJ, Ray CE, Johnson JL, Moore JB, Burch JM (2003) Cervical spine fracture patterns predictive of blunt vertebral artery injury. J Trauma Injury Infect Crit Care 55(5):811-813
9. Cothren CC, Biffl WL, Moore EE, Kashuk JL, Johnson JL (2009) Treatment for blunt cerebrovascular injuries. Arch Surg 144(7):685

10. Daou B, Hammer C, Mouchtouris N, Starke RM, Koduri S, Yang S, Jabbour P, Rosenwasser R, Tjoumakaris S (2017) Anticoagulation vs antiplatelet treatment in patients with carotid and vertebral artery dissection: a study of 370 patients and literature review. Neurosurgery 80(3):368-379

11. Drainer EK, Graham CA, Munro PT (2003) Blunt cervical spine injuries in Scotland 1995-2000. Injury 34(5):330-333

12. Esnault $\mathrm{P}$, Cardinale $\mathrm{M}$, Boret $\mathrm{H}$ et al (2017) Blunt cerebrovascular injuries in severe traumatic brain injury: incidence, risk factors, and evolution. J Neurosurg 127(1):16-22

13. Franz RW, Willette PA, Wood MJ, Wright ML, Hartman JF (2012) A systematic review and meta-analysis of diagnostic screening criteria for blunt cerebrovascular injuries. J Am Coll Surg 214(3): 313-327

14. Geddes AE, Burlew CC, Wagenaar AE, Biffl WL, Johnson JL, Pieracci FM, Campion EM, Moore EE (2016) Expanded screening criteria for blunt cerebrovascular injury: a bigger impact than anticipated. Am J Surg 212(6):1167-1174

15. Gennarelli TA, Wodzin E (2006) AIS 2005: a contemporary injury scale. Injury 37(12):1083-1091

16. Ghobrial GM, Amenta PS, Maltenfort M, Williams KA Jr, Harrop JS, Sharan A, Jallo J, Ratliff J, Prasad S (2014) Longitudinal incidence and concurrence rates for traumatic brain injury and spine injury - a twenty year analysis. Clin Neurol Neurosurg 123:174 180

17. Griessenauer CJ, Fleming JB, Richards BF et al (2013) Timing and mechanism of ischemic stroke due to extracranial blunt traumatic cerebrovascular injury. J Neurosurg 118(2):397-404

18. Grigorian A, Kabutey N-K, Schubl S, de Virgilio C, Joe V, Dolich M, Elfenbein D, Nahmias J (2018) Blunt cerebrovascular injury incidence, stroke-rate, and mortality with the expanded Denver criteria. Surgery 164(3):494-499

19. Grossman MD, Reilly PM, Gillett T, Gillett D (1999) National survey of the incidence of cervical spine injury and approach to cervical spine clearance in U.S. Trauma Centers. J Trauma Injury Infect Crit Care 47(4):684-690

20. Harrop JS, Sharan AD, Vaccaro AR, Przybylski GJ (2001) The cause of neurologic deterioration after acute cervical spinal cord injury. Spine 26(4):340-346

21. Heinänen $M$, Brinck T, Handolin L, Mattila VM, Söderlund T (2017) Accuracy and coverage of diagnosis and procedural coding of severely injured patients in the Finnish hospital discharge register: comparison to patient files and the Helsinki trauma registry. Scand J Surg 106(3):269-277

22. Hoffman JR, Wolfson AB, Todd K, Mower WR (1998) Selective cervical spine radiography in blunt trauma: methodology of the national emergency X-radiography utilization study (NEXUS). Ann Emerg Med 32(4):461-469

23. Hoffman JR, Mower WR, Wolfson AB, Todd KH, Zucker MI (2000) Validity of a set of clinical criteria to rule out injury to the cervical spine in patients with blunt trauma. N Engl J Med 343(2): 94-99

24. Holly LT, Kelly DF, Counelis GJ, Blinman T, McArthur DL, Cryer HG (2002) Cervical spine trauma associated with moderate and severe head injury: incidence, risk factors, and injury characteristics. J Neurosurg Spine 96(3):285-291

25. Jennett B, Bond M (1975) Assessment of outcome after severe brain damage. Lancet (London, England) 1(7905):480-484

26. Lauerman MH, Feeney T, Sliker CW, Saksobhavivat N, Bruns BR, Laser A, Tesoriero R, Brenner M, Scalea TM, Stein DM (2015) Lethal now or lethal later. J Trauma Acute Care Surg 78(6):10711075

27. Lindfors M, Vehviläinen J, Siironen J, Kivisaari R, Skrifvars MB, Raj R (2018) Temporal changes in outcome following intensive 
care unit treatment after traumatic brain injury: a 17-year experience in a large academic neurosurgical centre. Acta Neurochir 160(11): 2107-2115

28. Maas AIR, Hukkelhoven CWPM, Marshall LF, Steyerberg EW (2005) Prediction of outcome in traumatic brain injury with computed tomographic characteristics: a comparison between the computed tomographic classification and combinations of computed tomographic predictors. Neurosurgery 57(6):1173-1182 discussion 1173-82

29. Markus HS, Hayter E, Levi C et al (2015) Antiplatelet treatment compared with anticoagulation treatment for cervical artery dissection (CADISS): a randomised trial. Lancet Neurol 14(4):361-367

30. Mayberry JC, Brown CV, Mullins RJ, Velmahos GC (2004) Blunt carotid artery injury. Arch Surg 139(6):609

31. McNutt MK, Kale AC, Kitagawa RS et al (2018) Management of blunt cerebrovascular injury (BCVI) in the multisystem injury patient with contraindications to immediate anti-thrombotic therapy. Injury 49(1):67-74

32. Miyanji F, Furlan JC, Aarabi B, Arnold PM, Fehlings MG (2007) Acute cervical traumatic spinal cord injury: MR imaging findings correlated with neurologic outcome - prospective study with 100 consecutive patients. Radiology 243(3):820-827

33. Raj R, Siironen J, Kivisaari R, Kuisma M, Brinck T, Lappalainen J, Skrifvars MB (2013) Factors correlating with delayed trauma center admission following traumatic brain injury. Scand J Trauma Resusc Emerg Med 21(1):67

34. Shafafy R, Suresh S, Afolayan JO, Vaccaro AR, Panchmatia JR (2017) Blunt vertebral vascular injury in trauma patients: ATLS® recommendations and review of current evidence. J Spine Surg (Hong Kong) 3(2):217-225

35. Shahan CP, Magnotti LJ, Stickley SM, Weinberg JA, Hendrick LE, Uhlmann RA, Schroeppel TJ, Hoit DA, Croce MA, Fabian TC (2016) A safe and effective management strategy for blunt cerebrovascular injury. J Trauma Acute Care Surg 80(6):915-922

36. Stein DM, Boswell S, Sliker CW, Lui FY, Scalea TM (2009) Blunt cerebrovascular injuries: does treatment always matter? J Trauma Injury Infect Crit Care 66(1):132-144

37. Stiell IG, Wells GA, Vandemheen KL et al (2001) The Canadian Cspine rule for radiography in alert and stable trauma patients. J Am Med Assoc 286(15): 1841-1848

38. Thesleff T, Kataja A, Öhman J, Luoto TM (2017) Head injuries and the risk of concurrent cervical spine fractures. Acta Neurochir 159(5):907-914

39. Tobert DG, Le HV, Blucher JA, Harris MB, Schoenfeld AJ (2018) The clinical implications of adding CT angiography in the evaluation of cervical spine fractures. J Bone Joint Surg 100(17):14901495

40. Weber CD, Lefering R, Kobbe P, Horst K, Pishnamaz M, Sellei RM, Hildebrand F, Pape H-C, DGU T (2018) Blunt cerebrovascular artery injury and stroke in severely injured patients: an international multicenter analysis. World J Surg 42(7):2043-2053

41. Williams J, Jehle D, Cottington E, Shufflebarger C (1992) Head, facial, and clavicular trauma as a predictor of cervical-spine injury. Ann Emerg Med 21(6):719-722

42. Zetterberg H, Smith DH, Blennow K (2013) Biomarkers of mild traumatic brain injury in cerebrospinal fluid and blood. Nat Rev Neurol 9(4):201-210
Comments The aim of the present paper is to investigate on the relationship between blunt cerebrovascular injuries (BCVI), cervical spinal injuries (CSI) and outcome in severe traumatic injuries (TBI).

Among this special clinical category, the Authors withdrew a number of patients from the Helsinki Trauma Registry treated during 2015-2017 in a large level 1 trauma hospital. A multivariable logistic regression analysis was used and both functional mortality outcome was assessed at 6 months.

Among 255 patients with a cervical spine CT, 26 patients (10\%) had a CSI and of 194 patients with cervical CTangiography, 16 patients (8\%) had a BCVI. Four of the 16 BCVI patients had a BCVI-related brain infarction and 4 of the CSI patients had some form of spinal cord injury.

The Authors did not find any association between CSI and outcome and concluded that BCVI with concomitant TBI was an independent predictor of poor outcome in patients with severe TBI as well as no association between CSI and outcome after severe TBI was found.

Considerations 1) In the present paper no association between CSI and outcome of severe TBI was found. Nevertheless, postadmission neurological deterioration can be a dreaded complication of any spinal cord injury (SCI) since it can drive further respiratory and metabolic complications consistent with a TBI worsening (1).

2) When dealing with severe TBI, of which Diffuse Axonal Injury (DAI) represent one of the most challenging features, cerebral haemodynamic changes can occur. Hyperflow along with intracranial hypertension, variably responsive to barbiturate therapy, has been described in DAI patients by means of Transcranial Doppler Sonography (TCD), Flowmetry and Cerebral O2 Extraction (CEO2) driving to eventual intracranial (malignant) hypertension. The observation of the time course of the parameters studied allows to modify the pharmacological treatment and/or perform surgical decompression. In such circumstances it is possible to speculate on a possible "protective mechanism" operated by the secondary hyperflow condition against the potentially ischemic consequencies associated with CSI and carotid and vertebral arteries damage and the latter could also have a preventing effect against the evolution of hyperflow to hypoflow syndromes.

Further studies on such haemodynamic association are strongly advocated in order to better understand a "better prognosis" in this extreme isolated case.

\section{Massimiliano VISOCCHI}

Rome, Italy

\section{REFERENCES}

1) Visocchi M, Di Rocco F, Meglio M(2003) Subacute clinical onset of postraumatic myelopathy. Acta Neurochir (Wien Austria ) 145: 799 804.

2) Visocchi M, Chiaretti A, Genovese , Di Rocco F (2007) Haemodynamic pattern in children with postraumatic diffuse brain swelling. A preliminary study in 6 cases with neuroradiological features consistent with Diffuse Axonal Injury. Acta Neurochir (Wien) 149: 347 356.

Publisher's note Springer Nature remains neutral with regard to jurisdictional claims in published maps and institutional affiliations. 